\title{
Development of stability indicating method for quality assessment of African Albendazole tablets
}

\author{
Amine Hssaine ${ }^{1,2}$, Miloud El Karbane ${ }^{3}$, Mohamed Azougagh ${ }^{1}$, Imad Eddine Houti ${ }^{4}$, and Brahim Benaji ${ }^{1,2}$ \\ ${ }^{1}$ Biomedical Engineering and Pharmaceuticals Sciences Groupe Research - National Graduate School of Arts and Crafts (ENSAM)- \\ Mohammed V University Rabat, Morocco \\ ${ }^{2}$ Group of Bio-pharmaceutical and Toxicology Analysis- Laboratory of Pharmacology and Toxicology, Research Center of Drugs \\ Sciences -Faculty of Medicine and Pharmacy- Mohammed V University, Rabat, Morocco \\ ${ }^{3}$ Laboratory of Analytical Chemistry and Bromatology - Research Center of Drugs Sciences -Faculty of Medicine and Pharmacy- \\ Mohammed V University, Rabat, Morocco \\ ${ }^{4}$ Pharmacology and Toxicology group Research-Department of Biology-Faculty of Sciences Ain Chock-UH2-Casablanca, Morocco.
}

\begin{abstract}
In order to assess the quality of Albendazole tablets (Alb) sampled in three countries from West Africa, several physical and chemical tests were performed on tablets at normal conditions. A simple and economic HPLC method has been developed, validated and used for the simultaneous determination of Albendazole (Alb) content, as well as its impurities and the uniformity of its content. The stabilityindicating HPLC method was performed on a Symmetry $\mathrm{C}_{18}-5 \mu \mathrm{m} 250 \mathrm{~mm} \times 4.6 \mathrm{~mm}$ column with a gradient elution using a mobile phase composed of acetonitrile and sodium acetate buffer. The flow rate was set at $1 \mathrm{~mL} \cdot \mathrm{min}^{-1}$ and the eluent was monitored at $295 \mathrm{~nm}$. The method was validated for specificity, linearity, accuracy, precision, robustness and detection and quantification limits, in accordance with International Conference on Harmonisation Quality 2 (ICH Q2) guidelines. This method was performed on different Alb samples (originals and generics) products collected from Senegal, Niger and Mali. The obtained results showed that, the contents of the generic tablets from Niger and Mali comply with the United States Pharmacopeia (USP) monograph acceptance criteria. However, more than $20 \%$ of the generic tablets don't meet the USP monograph impurity limits. In conclusion, the described analytical method is simple, sensitive and accurate. Thus, it could be useful for manufacturing and quality control assays.
\end{abstract}

Key words: Quality, Stability, Albendazole tablets, monitoring, impurities.

\section{Introduction}

In developing countries, where antihelminthic drugs are mostly imported or manufactured locally as low cost generics, risks are high regarding the quality of these drugs, as they could be impacted in terms of the content of the active ingredient, as well as their purity, dissolution and bioavailability, thus impacting their therapeutic effectiveness [1].

The prescription of generic drugs is encouraged, but a rigorous vigilance should be implemented as most of these countries do not have the right competent authorities to ensure the quality of the drugs and avoid counterfeit preparations. Thus, quick, simple and effective standard controls must be developed and shaped to ensure the various quality aspects of these medicines [2].

Albendazole, methyl [5-(propilthio)1Hbenzimidazole-2-yl] carbamate (Figure 1) is a member of the benzimidazole compounds used as an anti-parasitic drug to treat a variety of worm infestations [3-5]. Albendazole is also widely used thanks to its broad activity spectrum. To be efficient, it should have the required physical and chemical qualities. The pharmaceutical form of Alb has been evaluated by several quality assessment techniques. It was quantified by liquid chromatography - Ultra Violet (UV) detector [6, 7], Reversed-Phase High Performance Liquid Chromatography (RP-HPLC) method with fluorescence detection $[8,9]$, electrochemical and spectrophotometric methods [10]. High performance liquid chromatographic process related to the separation and determination of impurities of Menbendazole, Fenbendazole and Albendazole in bulk drug was also studied [11].

In order to find more accurate and easy methods to control the quality of the marketed drugs, this study aimed to develop and validate an RP-HPLC stability indicating method for the simultaneous analysis of Alb dosage as an active pharmaceutical ingredient and Imp B and Imp C as related substances within normal conditions. The quality evaluation of Alb original and generic tablets marketed in Africa was also studied.<smiles>CCCSc1ccc2[nH]c(NC(=O)OC)nc2c1</smiles>

Fig.1. Chemical structure of Alb and its potential impurities (Imp B : Albendazole sulphoxide; and Imp C : Albendazole sulphone)

*Corresponding authors: hssaineamine@,gmail.combrahim.benaji@um5.ac.ma 


\section{Materials and methods}

\subsection{Chemicals and standards}

Alb (97.2 \%) was obtained from USP, Imp B (97.3\% Albendazole sulphoxide) and Imp C (99.5\% Albendazole sulphone) were supplied by Pfizer (Morocco).

Hydrochloric acid $(37 \%$, w/v), hydroxide sodium, peroxide $(30 \%, \mathrm{w} / \mathrm{w})$, acetate sodium, acetic acid and sulphuric acid $(85 \%$, w/w) were purchased from SigmaAldrich (Germany) and triethylamine was supplied by Merck KGaA (Germany). All these reagents were analytical grade. Methanol and acetonitrile are both HPLC grade and were purchased from Merck (Germany).The placebo, used in the analytical method validation, was prepared by mixing the following excipients used in different sampled market formulations (lactose, maize starch, povidone, microcrystalline cellulose, croscarmelose sodium, sunset yellow aluminum lake, sodium lauryl sulfate, saccharin sodium, vanilla flavoring, passion fruit flavoring, orange flavoring and magnesium stearate).

\subsection{Instruments}

The chromatographic system, used for the development and validation methods, consisted of Waters 2695 pump plus an auto sampler, and Waters 2998 photodiode-array detector (PDA). Data acquisition was performed by the Empower software. Binder climate chamber (USA) was used for photolytic degradation.

\subsection{Development of RP-HPLC method}

\subsubsection{Chromatography conditions}

Due to the differences of hydrophobic character between Albendazole and its known impurities, the simultaneous separation of the analytes under isocratic conditions; would take substantial time; therefore, a gradient elution was applied.

The chromatographic separation was performed on YMC Symmetry $C_{18}(5 \mu \mathrm{m} 250 \mathrm{~mm} \times 4.6 \mathrm{~mm})$ column at $35{ }^{\circ} \mathrm{C}$ using a constant flow rate of $1 \mathrm{~mL} / \mathrm{min}$ and a gradient elution with a buffer at $\mathrm{pH} 5.2$ and acetonitrile (Table 1). All the analytes were monitored at $295 \mathrm{~nm}$ by UV detection, and the injection volume was set at $50 \mu \mathrm{L}$.

Table 1: A gradient of mobile phase

\begin{tabular}{lll}
\hline Time (min) & Buffer & Acetonitrile \\
\hline $\mathbf{0}$ & 70 & 30 \\
$\mathbf{5}$ & 70 & 30 \\
$\mathbf{8}$ & 40 & 60 \\
$\mathbf{1 2}$ & 40 & 60 \\
$\mathbf{1 5}$ & 70 & 30 \\
\hline
\end{tabular}

\subsubsection{Solution preparations}

\subsubsection{Standard solutions preparations}

A standard solution, for the Albendazole assay, was prepared by dissolving appropriate amount $(25 \mathrm{mg})$ with $5 \mathrm{ml}$ mixture of sulphuric acid and methanol $(1: 99 ; \mathrm{v} / \mathrm{v})$ as diluent 1 to $50 \mathrm{~mL}$ volumetric flask and sonicated for about ten minutes, then completed with methanol as diluent 2 to obtain the concentration of $0.5 \mathrm{mg} / \mathrm{mL}$. A standard solution of impurities (mixture of Imp B and ImpC) at a concentration of $5 \mu \mathrm{g} / \mathrm{mL}$ was also prepared in same way.

\subsubsection{Assay, impurities and uniformity content sample solution preparations}

Twenty tablets $(400 \mathrm{mg})$ were weighed and the average weight was calculated. The tablets were powdered and a powder sample equivalent to $50 \mathrm{mg}$ of the active pharmaceutical ingredient (Albendazole) was transferred to $100 \mathrm{~mL}$ volumetric flask for the assay and for the impurities test, while the whole individual tablet was transferred to $100 \mathrm{~mL}$ volumetric flask for uniformity content. Approximately $10 \mathrm{~mL}$ diluent 1 was added and shaken. Finally, the solution was then diluted to $100 \mathrm{~mL}$ with diluent 2 and filtered through a $0.45 \mu \mathrm{m}$ pore size Rcellulose membrane filter. The filtrate was used as the sample solution.

\subsubsection{Validation of the assay method}

\subsubsection{Specificity}

The specificity of Albendazole, in the presence of its impurities namely imp-B, imp-C, degradation products and excipients, was determined by the developed HPLC method. Forced degradation studies were also performed on Albendazole to provide an indication of the stability and specificity properties of the proposed method. The stress conditions employed for degradation study includes light (carried out as per ICH Q1B), heat $\left(80^{\circ} \mathrm{C}\right)$, acid hydrolysis $(1 \mathrm{~N} \mathrm{HCl})$, base hydrolysis $(1 \mathrm{~N} \mathrm{NaOH})$ and oxidation $\left(5 \% \mathrm{H}_{2} \mathrm{O}_{2}\right)$. Thermal stress period was done for 2 days and photolytic degradation was carried out by exposing the sample to light for $240 \mathrm{~h}$ resulting in an overall illustration 1.2 million lux $\mathrm{h}$. whereas the time used for acid, base and peroxide hydrolysis the test period was $1 \mathrm{~h}, 1 \mathrm{~h}$ and $3 \mathrm{~h}$ respectively.

The heat study lasted 2 days, as for the luminosity, the sample was enlightened with 1.2 million Lux hours during the study period. Peak purity of stressed samples of Albendazole was checked by using 2996 Photo diode array detector of Waters Corporation, MA, USA.

\subsubsection{Precision}

The precision of the related substance method was checked by injecting six individual preparations of (50 $\mu \mathrm{g} / \mathrm{mL}$ ) Albendazole spiked with $0.75 \%$ each imp-B and imp-C. The Relative Standard Deviation (\%RSD) area of each imp-B and imp-C was calculated. Intermediate 
precision study was also determined by performing the same procedures on three different days.

\subsubsection{Linearity}

Linear calibration plots of the proposed method were obtained over concentration ranges of $24-700 \mu \mathrm{g} / \mathrm{mL}(24$, $120,240,400,500,580$, and $700 \mu \mathrm{g} / \mathrm{mL}$ ) for assay and of $2.5-7 \mu \mathrm{g} / \mathrm{mL}(2.5,4,5,6,7 \mu \mathrm{g} / \mathrm{mL})$ for impurities test. Each solution was prepared in triplicate.

\subsubsection{Accuracy}

The accuracy of the assay was evaluated in triplicate at five synthetic mixtures concentration levels, i.e. 25,75 , 100,125 and $150 \%$ of nominal analytical concentration ( $50 \mu \mathrm{g} / \mathrm{mL}$ of Albendazole) by spiking the placebo with Albendazole of known purity. At each concentration, three concentration levels were prepared and the percentage of recovery was calculated for each level.

The accuracy of the impurities method was evaluated in triplicate at $0.1,0.2,0.5,1$ and $1.2 \%$ by spiking the synthetic mixtures of nominal analytical concentration (50 $\mu \mathrm{g} / \mathrm{mL}$ of Albendazole) with impurities of known purity. The percentage of recoveries for impurities was calculated at each level.

\subsubsection{Limits of Detection (LOD) and Quantification (LOQ)}

The LOD and LOQ for all impurities and Albendazole were estimated at a signal-to-noise ratio of $3: 1$ and 10:1, respectively, by injecting a series of diluted solutions with known concentration. Precision study was also carried at the LOQ level by injecting six individual preparations of all impurities and Albendazole and calculating the \%RSD of the area. Accuracy at LOQ level was evaluated in triplicate for the nine impurities by spiking the impurities at the estimated LOQ level to test solution.

\subsubsection{Solution Stability and Mobile Phase Stability}

The stability of Albendazole and its impurities in solution was determined by leaving the spiked sample solution in a tightly capped volumetric flask at room temperature every $12 \mathrm{~h}$.

\section{Results and discussion}

\subsection{Validation of RP-HPLC method}

\subsubsection{Specificity}

Specificity of the RP - HPLC method, developed for the Alb, Imp B and Imp C, was investigated in order to obtain an indication of the possible interferences from the other components during routine analysis. The results in Figure 2 show that there are no interferences with Alb and its impurities peaks. Alb was observed to be well resolved from the degradation peaks (Imp-B and Imp C). Resolution value between Imp-C and Imp-B, and Alb and Imp-C were 8 and 40, respectively (Figure 2). Accordingly, chromatograms of the samples treated with acid, base, hydrogen peroxide, and thermal, showed clear separated peaks of pure Alb and its impurities (Figure 3).

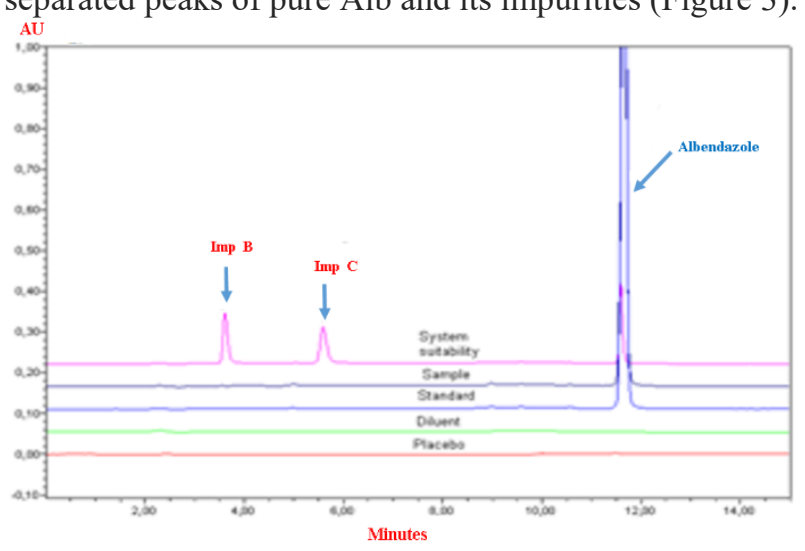

Fig.2. System suitability of validated method
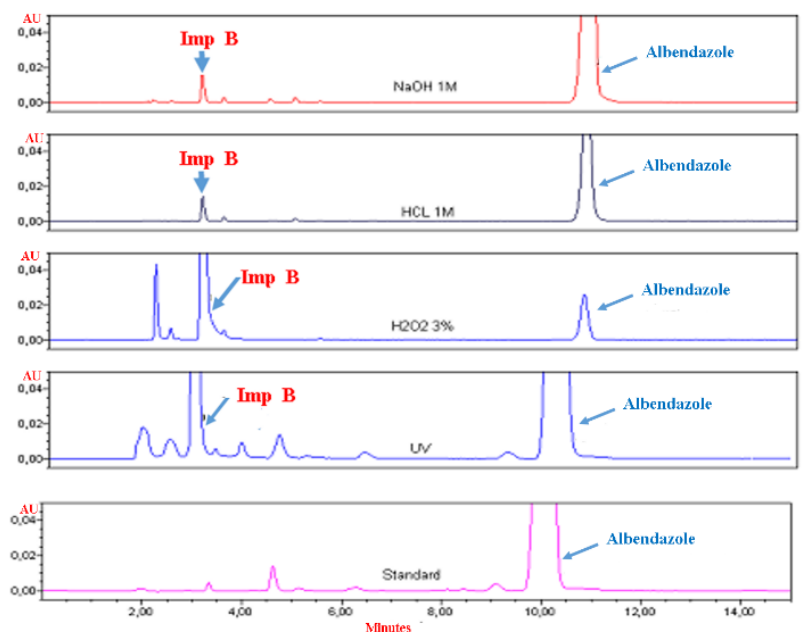

Fig.3. Forced degradation of a mixture of the fourteen drug products excipients for verification of specificity method

\subsubsection{Linearity}

Five calibration standards for $\mathrm{Alb}$ at high and low concentrations, Imp-B and Imp-C were prepared in order to evaluate the relationship between the area under the curve and the concentration. The linearity was evaluated in a concentration range of $240-700 \mu \mathrm{g} / \mathrm{mL}, 2.4-7.0$ $\mu \mathrm{g} / \mathrm{mL}, 2.4-7.0 \mu \mathrm{g} / \mathrm{mL}, 2.4-7.0 \mu \mathrm{g} / \mathrm{mL}$, respectively, covering the normal range of concentrations obtained when analyzing the content of Alb, Imp-B, Imp-C and unknown impurities percentage in the tablet form with $\mathrm{R}^{2}$ equal to $0.9993,0.9997$, and 0.9990 and 0.9991, respectively (Table 2).

Table 2 :Validation of RP - HPLC method for quantification of Alb and its impurities 
Accuracy (Recovery \% )

\begin{tabular}{|c|c|c|c|c|}
\hline Level & $\begin{array}{l}\text { Alb at high } \\
\text { concentration } \\
\text { for assay }\end{array}$ & $\begin{array}{c}\text { Alb at low } \\
\text { concentration for assay } \\
\text { of other impurities }\end{array}$ & Imp B & Imp C \\
\hline 1 & 100.67 & 101.38 & 99.80 & 102.02 \\
\hline 2 & 102.38 & 98.61 & 99.72 & 99.57 \\
\hline 3 & 100.05 & 98.80 & 100.56 & 99.92 \\
\hline 4 & 101.36 & 99.78 & 100.47 & 100.82 \\
\hline 5 & 99.78 & 99.72 & 99.92 & 100.76 \\
\hline Mean $(\%)$ & 100.48 & 99.65 & 100.09 & 100.61 \\
\hline Confidence Interval & $99.97-101.73$ & $98.89-100.43$ & $99.82-100.37$ & $99.80-101.44$ \\
\hline \multicolumn{5}{|c|}{ Repeatability and Intermediate Precision (RSD \%) } \\
\hline Day 1 & 1.57 & 0.57 & 0.51 & 0.70 \\
\hline Day 2 & 0.70 & 0.89 & 0.89 & 0.63 \\
\hline Day 3 & 0.37 & 0.30 & 0.17 & 0.23 \\
\hline Intra - day & 1.01 & 0.61 & 0.61 & 0.72 \\
\hline Inter-day & 1.30 & 0.65 & 1.93 & 1.72 \\
\hline \multicolumn{5}{|l|}{ Linearity } \\
\hline Slope & 24568087 & 57769632.87 & 58967285.42 & 57075093.4 \\
\hline Intercept & 1433668 & -2119.94 & 9024.23 & -1452.763238 \\
\hline $\begin{array}{l}\text { Correlation Coefficient } \\
\left(\mathrm{R}^{2}\right)\end{array}$ & 0.9993 & 0.9991 & 0.9997 & 0.9990 \\
\hline Range $(\mu \mathrm{g} / \mathrm{mL})$ & {$[240-700]$} & {$[2.4-7.0]$} & {$[2.4-7.0]$} & {$[2.4-7.0]$} \\
\hline \multicolumn{5}{|l|}{ LOQ and LOD } \\
\hline LOQ $(\mu \mathrm{g} / \mathrm{mL})$ & NA & 2.4 & 2.4 & 2.4 \\
\hline LOD $(\mu \mathrm{g} / \mathrm{mL})$ & NA & 0.8 & 0.8 & 0.8 \\
\hline
\end{tabular}

\subsubsection{Precision}

The repeatability of the method was tested by analyzing six replicate samples of $500 \mu \mathrm{g} / \mathrm{mL}$ of $\mathrm{Alb}$ as a high concentration, $5 \mu \mathrm{g} / \mathrm{mL}$ of Alb as a low concentration, 5 $\mu \mathrm{g} / \mathrm{mL}$ of Imp B and $5 \mu \mathrm{g} / \mathrm{mL}$ of Imp C; the relative standard deviations were $1.01,0.61,0.72$ and $0.61 \%$, respectively. The intermediate precision (Inter- days) of the same concentrations of Alb high concentration, Alb low concentration, Imp-B and Imp-C Where evaluated over three days, by performing six measurements each day. The relative standard deviations are 1.30, 0.65, 1.72 and $1.93 \%$, respectively (Table 2 ).

\subsubsection{Accuracy}

The method accuracy was determined by spiking placebobased solutions with Alb high concentration, Alb low concentration, Imp-B and Imp-C to determine recovery at three levels with concentrations ranging from approximately 80 to $120 \%$. Recoveries were $100.48 \%$, $99.65 \%, 100.09 \%$ and $100.61 \%$ for Alb at high concentration, Alb at low concentration Imp-B and for Imp-C respectively (Table 2 ).
The limit of detection is the smallest quantity of the targeted substance that can be detected, but not accurately quantified in the sample. The lower limit of quantitation (LOQ) is the smallest quantity of the targeted substance in the sample that can be assayed under experimental conditions with well-defined accuracy. By applying this computation method, the LOD of Alb at low concentration, Imp-B and Imp-C were equal to $0.8 \mu \mathrm{g} / \mathrm{mL}$, while their LOQ were $2.4 \mu \mathrm{g} / \mathrm{mL}$ (Table 2).

\subsection{Quality assessment of Alb tablet}

The dosage results of Alb and impurity contents in the tablets marketed in Africa were reported (Table 3). The content of Alb in the tablets was found to be within normal range of $\pm 5 \%$ for all products except product 4 . In addition, the uniformity dosage unite was according to USP norm $85 \%-115 \%$ [12], (results are not shown). However, the impurity content of Imp B and Imp C are higher than the limits set by the USP due to the API degradation.

\subsection{5 $L O Q$ and $L O D$}


Table 3: Assay of Alb content in tablet and impurities profile at normal conditions

\begin{tabular}{|l|l|c|c|c|c|}
\hline \multirow{3}{*}{ Countries } & N (Manufacture) & Assay \% & RSD \% & Imp B & Imp C \\
& & & & & \\
\hline Senegal & P1 (South Afr.) & 99.08 & 0.39 & 0.27 & 0.13 \\
& P2 (India) & 102.33 & 0.51 & 0.57 & 0.15 \\
& P3 (Senegal) & 99.81 & 0.66 & 0.20 & 0.14 \\
& P4(China) & 94.12 & 0.35 & 0.18 & 0.08 \\
\hline Niger & P5 (South Afr.) & 98.35 & 0.55 & 0.07 & 0.12 \\
& P6 (India) & 95.48 & 0.23 & 0.15 & 0.10 \\
& P7 (Togo) & 101.30 & 0.61 & 0.10 & 0.11 \\
& P8 (India) & 103.12 & 1.43 & 0.10 & 0.10 \\
& P9 (India) & 100.32 & 0.59 & 0.09 & 0.11 \\
& & 99.56 & 0.40 & 0.21 & 0.09 \\
& P10 (South Afr.) & 98.33 & 0.18 & 0.16 & 0.07 \\
& P11 (South Afr.) & & & & \\
& P12 (India) & & & & \\
& P13 (India) & 101.10 & 0.90 & 0.25 & 0.13 \\
& P14 (India) & 100.65 & 0.42 & 0.35 & 0.21 \\
& & 101.77 & 1.01 & 0.50 & 0.29 \\
\hline
\end{tabular}

\section{Conclusion}

Drugs and health products undergo scheduled controls, based on multiple criteria risk analysis such as the onset probability of a quality defect, the nature of substantially associated adverse events and the level of exposure to the population. This quality control study is a risk analysis; in order to have an independent expertise and strengthen the safety of health products for the patients. Because of the globalization of markets, the emergence of falsifications and the occurrence of new infectious agents; it is important to acquire strong quality control units within the laboratories. The risks quality management is now a statutory requirement. The developed stability indicating HPLC method was used for the evaluation of the African Albendazole tablets quality, including the purity, the assay and the stability. It should, suitably be used to ensure the quality of the products and focus efforts and the qualified resources where the risks are higher. Thus, the risks will be lower for the patients. It also enables to determine the manufacturing controls suitable levels leading to efficiency.

\section{References:}

1. WHO. Prevention and control of schistosomiasis and soil-transmitted helminthiasis. Geneva: World Health Organization; (2002)

2. WHO. Report of the WHO Informal Consultation on the Use of Chemotherapy for the Control of
Morbidity Due to Soil-Transmitted Nematodes in Humans, Geneva, 29 April to 1 May 1996, Geneva, World Health Organization, (1996) (document WHO/ CTD/SIP|96 P)

3. G. Navarette-Vàzquez, L. Yépez, A. HernàndezCampos, A. Tapia, F. Hernández-Luis, R. Cedillo, J. González, A. Martínez-Fernández, M. MartínezGrueiro, R. Castillo, Synthesis and antiparasitic activity of Albendazole and mebendazole analogues, Bioorg\& Med Chem,11, 4615-4622 (2003)

4. J. Horton,Albendazole: a review of anthelmintic efficacy and safety in humans, Parasitology,121, S113-S132 (2000)

5. J. Horton,Albendazole for the treatment of echinococcosis, Fund. \&Clin. Pharm, 17, 205212(2003)

6. N.N.A. Okine, J.S.K. Ayim,S.K.K. wakye, Analysis of Albendazole by HPLC method. West Afr. J. of Pharm., 13, 41-44 (1999)

7. P. Chiap, B. Evrard, M.A. Bimazubute, P. de Tullio, Ph. Hubert,L.Delattre, J. Crommen, Determination of Albendazole and its main metabolites in ovine plasma by liquid chromatography with dialysis as an integrated sample preparation technique, J. of Chroma. A, 870, 121-134(2000) 
8. G. C. Batzias,G. A. Delis, Reversed-phase liquid chromatographic method with fluorescence detection for the simultaneous determination of Albendazolesulphoxide, Albendazolesulphone and Albendazole2-aminosulphone in sheep plasma, J. of Chroma. B, 805, 267-274 (2004)

9. G.C. Batzias, E. Theodosiadou, G.A. Delis, Quantitative determination of Albendazole metabolites in sheep spermatozoa and seminal plasma by liquid chromatographic analysis with fluorescence detection, J. of Pharm. and Biom. Anal, 35, 191-120 (2004)

10. A. L. Santos, R. M. Takeuchi, N. R. Stradiotto, Electrochemical, Spectrophotometric and LiquidChromatographic Approaches for Analysis of Tropical Disease Drugs. Current Pharmaceutical Analysis, 5, 69-88 (2009)

11. A. R. Gomes, V. Nagaraju, High performance liquid chromatographic separation and determination of the process related impurities of menbendazole, fenbendazole and Albendazole in bulk drug, J. Pharm. and Biom. Anal.,26, 919 - 927 (2001)

12. United States Pharmacopeia, United States Pharmacopeia Convention. Rockville, MD: USP. (2009) 